\title{
MODELLING OF KINETICS OF AUSTENITE-TO-ALLOTRIOMORPHIC FERRITE TRANSFORMATION IN 0.37C-1.45Mn-0.11V MICROALLOYED STEEL
}

\author{
C. CAPDEVILA, F. G. CABALLERO, and C. GARCÍA DE ANDRÉS
}

\begin{abstract}
The present paper is concerned to the theoretical and experimental study of the growth kinetics of allotriomorphic ferrite in medium carbon vanadium-titanium microalloyed steel. A theoretical model is presented in this work to calculate the evolution of austenite-to-allotriomorphic ferrite transformation with time at a very wide temperature range. At temperatures above eutectoid temperature, where allotriomorphic ferrite is the only austenite transformation product, softimpingement effect should be taken into account in the modeling. In that case, the Gilmour et al. analysis reliably predicts the progress of austenite-to-allotriomorphic ferrite transformation in this steel. By contrast, since pearlite acts as a carbon sink, the carbon enrichment of austenite due to

C. CAPDEVILA, Research Associate, F.G. CABALLERO, Research Associate, and C. GARCÍA DE ANDRÉS, Senior Research Fellow, are in the Department of Physical Metallurgy, Centro Nacional de Investigaciones Metalúrgicas (CENIM), Consejo Superior de Investigaciones Científicas (CSIC), Avda. Gregorio del Amo, 8, 28040 Madrid, Spain. C.CAPDEVILA currently is at the Department of Materials Science and Metallurgy, University of Cambridge, Pembroke Street, Cambridge CB2 3QZ, UK
\end{abstract}


the previous ferrite formation is avoided, and carbon concentration in austenite far from the $\alpha / \gamma$ interface remains the same as the overall carbon content of the steel. Hence, soft-impingement effect should be neglected, and allotriomorphic ferrite is considered to grow under a parabolic law. Therefore, assumption of a semi-infinite extent austenite with constant boundary conditions is suitable for the kinetics of the isothermal decomposition of austenite. An excellent agreement (higher than $93 \%$ in $\mathrm{R}^{2}$ ) has been obtained between experimental and predicted values of volume fraction of ferrite in all the studied range of temperature.

\section{INTRODUCTION}

The term allotriomorphic means that the phase is crystalline in internal structure but not in outward form. It implies that the limiting surfaces of the crystal are not regular and do not display the symmetry of its internal structure ${ }^{[1-3]}$. Allotriomorphic ferrite which nucleates at the prior austenite grain boundaries tends to grow along the austenite grain boundaries at a rate faster than in the normal direction to the boundary plane, so that its shape is strongly influenced by the presence of the grain boundaries and hence does not necessarily reflect its internal symmetry. Of course, allotriomorphic ferrite does not need to form just at austenite grain boundaries, but it invariably does so, presumably because there are no others two-dimensional heterogeneous nucleation sites in austenite more suitable ${ }^{[4]}$.

In the two last decades, many researchers have created models for the austenite-toallotriomorphic ferrite transformation based on thermodynamic and fundamental phase transformation theory for a wide range of steels ${ }^{[5-6]}$. Unemoto et al. ${ }^{[7]}$ developed a methodology to simulate the allotriomorphic ferrite transformation under isothermal conditions. The volume 
fraction of allotriomorphic ferrite was computed at every temperature using Cahn's theory for grain boundary nucleation kinetics ${ }^{[8]}$. Likewise, Hillert-Staffanson regular solution model ${ }^{[9]}$ was used to calculate thermodynamic data for several alloying elements under paraequilibrium conditions. Classical nucleation theory was applied for allotriomorphic ferrite, followed by parabolic growth kinetics. Finally, Lee et al. ${ }^{[10]}$ developed a mathematical model for the allotriomorphic ferrite transformation in $\mathrm{Nb}$ microalloyed steels, considering a Fe-C-Mn-Nb system. They used the Hillert-Staffanson model to determine the austenite and ferrite carbon content at the interface and the free energy of the transformation under local and paraequilibrium conditions.

Recent works have demonstrated that medium carbon microalloyed forging steels with acicular ferrite microstructure can be manufactured at industrial scale ${ }^{[11-15]}$. The main interest of this microstructure lies in the good combination of mechanical properties that presents as compared with bainite and especially with ferritic-pearlitic microstructures. In those steels, acicular ferrite is always formed after the growth of allotriomorphic ferrite and pearlite. As a consequence, acicular ferrite transformation is inevitably influenced by previous allotriomorphic ferrite formation. The role of the allotriomorphic ferrite to promote the formation of acicular ferrite to the detriment of bainite has been reported in previous works ${ }^{[16-19]}$. Thus, the amount of acicular ferrite increases as allotriomorphic ferrite is present along the austenite grain boundaries of medium carbon microalloyed steels. Therefore, a deep understanding of the decomposition of austenite in allotriomorphic ferrite is needed in order to control the total amount of acicular ferrite present in the microstructure.

The present paper is concerned to the theoretical and experimental description of the growth kinetics of allotriomorphic ferrite in medium carbon vanadium-titanium microalloyed steel in a very wide temperature range. The theoretical model presented in this work allows to calculate the 
evolution of austenite-to-allotriomorphic ferrite transformation with time at temperatures at which allotriomorphic ferrite is or is not the only austenite decomposition product. This work is the continuation of previous authors' study ${ }^{[20]}$.

\section{EXPERIMENTAL}

The chemical composition of the steel studied is presented in Table I. The material was supplied in the form of $50 \mathrm{~mm}$ square bars, obtained by conventional casting to a square ingot (2500 kg) and hot rolling to bar. Cylindrical dilatometric test pieces of $2 \mathrm{~mm}$ in diameter and 12 $\mathrm{mm}$ in length were machined parallel to the rolling direction of the bar.

Table I. Chemical composition (wt - \%) of the steel

\begin{tabular}{ccccccccc}
\hline $\mathrm{C}$ & $\mathrm{Si}$ & $\mathrm{Mn}$ & $\mathrm{Cr}$ & $\mathrm{Al}$ & $\mathrm{Ti}$ & $\mathrm{V}$ & $\mathrm{Cu}$ & $\mathrm{Mo}$ \\
\hline 0.37 & 0.56 & 1.45 & 0.04 & 0.024 & 0.015 & 0.11 & 0.14 & 0.025 \\
\hline
\end{tabular}

The isothermal decomposition of austenite has been analyzed by means of an Adamel Lhomargy DT1000 high-resolution dilatometer described elsewhere ${ }^{[21]}$. The incubation time of allotriomorphic ferrite, or minimum time at which it is possible to find some allotriomorphs nucleated on the austenite grain boundary, is experimentally determined from dilatometric curves obtained during the isothermal decomposition of austenite. The change in length of the specimen is transmitted via amorphous silica pushrod. These variations are measured by a linear variable differential transformer (LVDT) sensor in a gas-tight enclosure enabling testing under vacuum or an inert atmosphere with an accuracy lower than 0.1 micron and the dilatometric curve is monitored along the thermal cycle with the help of a computer assisted electronic device. 
Experimental validation of the allotriomorphic ferrite formation kinetics model developed in this work was carried out using the heating and cooling devices of the above mentioned dilatometer. The heating device consists of a very low thermal inertia radiation furnace. The power radiated by two tungsten filament lamps is focussed on the specimen by means of a bi-elliptical reflector. The temperature is measured with a $0.1 \mathrm{~mm}$ diameter Chromel - Alumel (Type K) thermocouple welded to the specimen. Cooling is carried out by blowing a jet of helium gas directly onto the specimen surface. The helium flow rate during cooling is controlled by a proportional servovalve. These devices ensure an excellent efficiency in controlling the temperature and holding time of isothermal treatments and as well as fast cooling in quenching processes.

As it is well known, prior austenite grain size (PAGS) exerts an important influence on the decomposition of austenite ${ }^{[22-23]}$. The PAGS parameter directly affects the growth kinetics of allotriomorphic ferrite obtained by isothermal decomposition of the austenite. But, there is no influence on the nucleation time of this phase ${ }^{[24-25]}$. In this sense, austenitisation conditions were fixed to avoid the influence of the austenite grain size on the kinetics of allotriomorphic ferrite formation and to study specifically the effect of the isothermal decomposition temperature on the growth kinetics of this phase. Since the growth rate of allotriomorphs is higher the finer the PAGS, a coarse PAGS of $76 \mu \mathrm{m}$ was selected to make easier the experimental study of the growth kinetic of allotriomorphic ferrite. Thus, specimens were austenitised at $1523 \mathrm{~K}$ for $1 \mathrm{~min}$ and subsequently isothermally transformed at $973 \mathrm{~K}, 913 \mathrm{~K}$ and $873 \mathrm{~K}$ during different times. In order to freeze the microstructure at those temperatures, specimens were quenched to room temperature by helium gas flow at a cooling rate of $200 \mathrm{~K} / \mathrm{s}$.

Specimens were polished in the usual way for metallographic examination. Nital - 2pct etching solution was used to reveal the ferrite microstructure by optical microscopy. The PAGS 
was estimated on micrographs by counting the number of grains intercepted by straight lines long enough to yield at least fifty intercepts in total. The effects of a moderately non-equiaxial structure may be eliminated by counting the intersections of lines in four or more orientations covering all the observation fields with an approximately equal weight ${ }^{[26]}$. Moreover, the volume fraction of allotriomorphic ferrite $\left(V_{\alpha}\right)$ was statistically estimated by a systematic manual point counting procedure ${ }^{[26]}$ A grid superimposed on the microstructure provides, after a suitable number of placements, an unbiased statistical estimation of the $V_{\alpha}$.

The austenite-to-allotriomorphic ferrite $\left(A e_{3}\right)$ and austenite-to-pearlite $\left(A e_{1}\right)$ critical temperatures were experimentally determined by dilatometric and metallographic analysis. Initially, both temperatures were estimated from a dilatometric curve obtained by continuous cooling at a rate of $0.05 \mathrm{~K} / \mathrm{s}$. This is the rate normally used for considering quasi-equilibrium conditions $^{[27]}$. Subsequently, those critical points were verified by interrupted cooling by quenching tests and metallographic examination of the obtained microstructure. Figure 1 shows both critical temperatures marked on the dilatometric curve in degrees centigrade $\left(\mathrm{Ae}_{3}=1013 \mathrm{~K}\right.$ and $\left.A e_{1}=943 \mathrm{~K}\right)$.

The equilibrium volume fraction of allotriomorphic ferrite $(\mathrm{Ve})$ formed during the isothermal decomposition of austenite at each temperature tested was again determined by a combination of dilatometric and metallographic analysis. For the three studied temperatures, the dilatometric curve (relative change of length $(\Delta L / L o)$ versus time $(t))$ reaches saturation at holding times lower than $10 \mathrm{~h}$. Then, isothermal heat treatments for $10 \mathrm{~h}$ allowed to measure $(\mathrm{Ve})$ at the three studied temperatures. Figure 2 shows the quenching microstructures obtained after the complete isothermal decomposition of austenite at 973, 913 and $873 \mathrm{~K}$. Table II lists the experimental 
values of $\mathrm{Ve}$. As this table shows, for temperatures below $A e_{1}$, the total amount of allotriomorphic ferrite decreases as isothermal temperature decreases.

\section{Table II. Equilibrium allotriomorphic ferrite volume fraction}

\begin{tabular}{cc}
\hline Temperature, $\mathrm{K}$ & $\mathrm{Ve}, \%$ \\
\hline 973 & 23 \\
\hline 913 & 28 \\
\hline 873 & 18 \\
\hline
\end{tabular}

\section{RESULTS AND DISCUSSION}

\section{A. Incubation time of allotriomorphic ferrite}

Lange et al. ${ }^{[28]}$ proposed a model to calculate the classical nucleation rate of allotriomorphic ferrite based on a traditional disk-shaped 'pillbox' nucleus. In that case, the incubation time for allotriomorphic ferrite is estimated as:

$$
\tau=\frac{12 k_{B} T a^{4} \sigma_{\alpha \gamma}}{D_{C}^{\gamma} \bar{x} v_{\alpha}^{2} \Delta G_{v}^{2}}
$$

where $k_{B}$ is Boltzmman constant; $D_{C}^{\gamma}$ is the diffusivity of carbon in austenite; $v_{\alpha}$ is the volume of an atom of iron in ferrite; $a$ is the average of the lattice parameters of both phases, ferrite and austenite; $\bar{X}$ is the average carbon content in mole fraction; $\Delta G_{v}$ is the volume free energy 
change associated to the formation of the nucleus; $\sigma_{\alpha \gamma}$ is the interfacial energy of a disorder ferrite; and $T$ is the isothermal temperature.

The theoretical determination of $D_{C}^{\gamma}$, due to Siller and McLellan ${ }^{[29]}$ and reviewed by Bhadeshia $^{[30]}$, considers both the kinetic and equilibrium thermodynamic behavior of carbon in austenite. Calculations of $D_{C}^{\gamma}$ takes also into account the concentration dependence of the activity of carbon in austenite, and the repulsive interactions between the nearest neighboring carbon atoms located in octahedral interstitial sites. Thus, the diffusivity $D_{C}^{\gamma}$ is calculated by two factors: one of them is a concentration dependent factor and the other one is independent

$$
D_{C}^{\gamma}=\xi(\theta) \frac{k_{B} T}{h}\left(\frac{\lambda^{2}}{3 \gamma_{m}}\right) \exp \left\{-\frac{\Delta G^{*}}{k_{B} T}\right\}
$$

where $\xi(\theta)$ is the carbon concentration dependent factor obtained according to Bhadeshia's calculations ${ }^{[30]}$ and takes values listed in Table III; $\Delta G^{*}$ is the activation free energy for diffusion which is independent of composition and temperature; $\gamma_{m}$ is an activity coefficient assumed constant; $\lambda$ is the distance between the $\{002\}$ austenite planes and $h$ is the Planck's constant. Bhadeshia ${ }^{[30]}$ found that $\Delta G^{*} / k_{B}=21230 \mathrm{~K}$ and $\ln \left(\gamma_{m} / \lambda^{2}\right)=31.84$. The values of $D_{C}^{\gamma}$ for temperatures ranging from $973 \mathrm{~K}$ to $873 \mathrm{~K}$ are also listed in Table III.

The volume free energy change, $\Delta G_{v}$, in equation (1) has been calculated with the help of commercial software denominated MTDATA ${ }^{[31]}$, which contains a large and rigorously evaluated thermodynamic database. $\Delta G_{v}$ calculations take into account the effect of all the alloying elements in the decomposition of austenite. The values obtained for the studied steels are listed in 
Table III. The value of $a$ in equation (1) has been calculated considering the influence of different alloying elements on ferrite and austenite lattice parameters. The ferrite lattice parameter has been calculated using the relations given by Bhadeshia ${ }^{[32]}$, whereas the austenite lattice parameter has been calculated as reported by Ridley et al. ${ }^{[33]}$ and Dyson and Holmes ${ }^{[34]}$. The values of $a$ at temperatures ranging from $973 \mathrm{~K}$ to $873 \mathrm{~K}$ are listed in Table III. Likewise, a $\sigma_{\alpha \gamma}$ value of 0.705 $\mathrm{J} \mathrm{m}^{-2}[28,35]$ and $v_{\alpha}=8.785 \times 10^{-30} \mathrm{~m}^{3}{ }^{[36]}$ have been considered in equation (1) for $\tau$ calculations.

Table III. Calculated values of $\xi(\theta), D_{C}^{\gamma}, \Delta G_{v}$, and $a$ parameters

\begin{tabular}{|c|c|c|c|c|}
\hline $\mathrm{T}, \mathrm{K}$ & $\xi(\theta)$ & $D_{C}^{\gamma} \times 10^{-14}, \mathrm{~m}^{2} \mathrm{~s}^{-1}$ & $\Delta G_{v} \times 10^{7}, \mathrm{~J} \mathrm{~m}^{-3}$ & $a \times 10^{-10}, \mathrm{~m}$ \\
\hline 973 & 0.009 & 30.9 & -0.96 & 3.306 \\
\hline 913 & 0.016 & 12.0 & -3.68 & 3.302 \\
\hline 873 & 0.023 & 5.8 & -5.94 & 3.300 \\
\hline
\end{tabular}

The experimental determination of incubation time has been carried out by dilatometry and metallography. The experimental incubation time is defined as the minimum time at which it is possible to find some allotriomorphs nucleated on the austenite grain boundary. A detailed analysis of the dilatometric curve associated to the isothermal decomposition of austenite (Figure 3) allows to determine an interval of time, $\Delta t$, in which it is more likely to find the incubation time. With the aim of carrying out an accurate determination, samples were isothermally treated at different holding time within the $\Delta t$ interval and immediately quenched. A metallographic analysis of those samples determined the incubation time at which some allotriomorphs appear in the microstructure. Figure 4 shows an example of a microstructure with allotriomorphs at the initial stage of their formation. 
Figure 5 shows a comparison between calculated (dashed line) and experimental (points) incubation time values for the studied steel. From this figure can be concluded that calculated results for the incubation time are in good agreement with the predicted values from calculations proposed in this work.

\section{B. Kinetics of austenite-to-allotriomorphic ferrite transformation}

After nucleation occurs at the austenite grain boundaries, the thin layers of ferrite that decorate the austenite grain boundaries thicken at a rate controlled mainly by the diffusion of carbon in the austenite ahead of the advancing ferrite/austenite interface ${ }^{[37]}$. The half-thickness of the allotriomorphs, $Z$, is generally calculated under parabolic growth conditions according to the following expression ${ }^{[38]}$ :

$$
Z=\alpha_{1} t^{1 / 2}
$$

where $\alpha_{1}$ is the one - dimensional parabolic growth rate constant and $t$ is the growth time. The value of $\alpha_{1}$ can be obtained by numerical solution from the equation ${ }^{[39]}$ :

$$
\alpha_{1} \exp \left(\frac{\alpha_{1}^{2}}{4 D_{C}^{\gamma}}\right) \operatorname{erfc}\left(\frac{\alpha_{1}}{2 \sqrt{D_{C}^{\gamma}}}\right)=2\left(\frac{D_{C}^{\gamma}}{\pi}\right)^{1 / 2} \frac{\bar{C}-C^{\gamma \alpha}}{C^{\gamma \alpha}-C^{\alpha \gamma}}
$$

where $D_{C}^{\gamma}$ is the diffusivity of carbon in austenite, $\bar{C}$ is the overall carbon content, $C^{\gamma \alpha}$ is the austenite solute content at the interface, and $C^{\alpha \gamma}$ is the ferrite solute content at the interface. 
According to Bhadeshia ${ }^{[40]}$, the consideration of paraequilibrium is a good approach for the kinetics of this transformation. In that case, partitioning of substitutional solute atoms does not have time to occur and the adjoining phases have identical $\mathrm{X} / \mathrm{Fe}$ atom ratios, where $\mathrm{X}$ represents the substitutional solute elements. Then, the substitutional lattice is configurationally frozen, but interstitial solutes such as carbon are able to partition and attain equilibration of chemical potential in both phases. Hence, the values of $C^{\gamma \alpha}$ and $C^{\alpha \gamma}$ in Eqn. (4) refer to carbon concentrations and they were calculated according to the procedure reported by Shiflet et al. ${ }^{[41]}$. $C^{\gamma \alpha}$ and $C^{\alpha \gamma}$ values as well as $\alpha_{1}$ values are listed in Table IV.

Table IV. Calculated values of $C^{\alpha \gamma}, C^{\gamma \alpha}$ and $\alpha_{1}$

\begin{tabular}{cccc}
\hline T, K & $C^{\alpha \gamma}$, wt. \% & $C^{\gamma \alpha}$, wt. $\%$ & $\alpha_{1} \times 10^{-7}, \mathrm{~m} \mathrm{~s}^{-1 / 2}$ \\
\hline 973 & 0.014 & 0.500 & 2.88 \\
913 & 0.016 & 0.903 & 4.85 \\
873 & 0.016 & 1.195 & 4.76 \\
\hline
\end{tabular}

\section{Kinetics of allotriomorphic ferrite formation at $T>A e_{1}$}

At temperatures where allotriomorphic ferrite is the only austenite decomposition product, overlapping of carbon diffusion gradients occurs due to allotriomorphs growing from opposite sides of a grain. This effect, known as soft-impingement, should be taken into account in the modeling of the growth kinetics of allotriomorphic ferrite. Soft impingement is considered in the model using an analytical treatment based on work by Gilmour et al. ${ }^{[42]}$. 
The one-dimensional growth of planar grain boundary allotriomorphs from opposite sides of an austenite grain is illustrated schematically in Figure 6(a). This process may be considered in two stages. The first stage involves a parabolic growth from both sides of the grain according to the assumption that austenite has a semi-infinite extent with constant boundary conditions. In this stage, the carbon concentration in austenite far from the $\alpha / \gamma$ interface remains the same as the overall carbon content of the steel. During the second stage, soft-impingement occurs, the growth rate considerably decreases and the austenite is considered to have a finite extent beyond the $\alpha / \gamma$ interface. Therefore, the carbon concentration in the center of the austenite grain is given by balancing the amount of carbon enrichment of the austenite against the corresponding depletion of the ferrite ${ }^{[40,43]}$. Figure $6(\mathrm{~b})$ shows a scheme of the carbon concentration profiles in austenite (dark lines) during the soft impingement stage. The carbon concentration in ferrite has been considered constant and negligibly small $\left(C^{\alpha \gamma}=0\right)$ (dark in $\mathrm{X}$-axis). It is assumed that $\alpha / \gamma$ interface moves in $z$ direction normal to the interface plane, and austenite is considered to have a finite size $L$ in that direction. The position of the interface at any time $t$ is defined by $z=Z$, being $Z=0$ at $t$ $=0$. In this initial state, the carbon concentration in the austenite is uniform and corresponds to the overall carbon composition $(\bar{C})$. The position of the interface at the onset of soft impingement is defined by $z=Z_{1}$ and $t=t_{1}$. At that moment, carbon concentration rises at every point in the austenite located ahead of the interface. At a subsequent stage $\left(z=Z_{2}\right.$ and $\left.t=t_{2}\right)$, the concentration of carbon in the center of the austenite grain increases from $\bar{C}$ to $C_{L}$. Finally, when $z=Z_{3}$ and $t=t_{3}$, the carbon content in austenite is uniform and equal to that of austenite in the $\alpha / \gamma$ interface $\left(C^{\gamma \alpha}\right)$.

At the onset of the soft impingement when the position of the interface is $z=Z_{1}$, the carbon enrichment of the austenite is equal to the amount of carbon removed from the ferrite. Therefore, 
according to Figure 6(b), the mass balance allows to calculate the position of the interface $Z_{1}$ which is given by:

$$
Z_{1}=\frac{L\left(C^{\gamma \alpha}-\bar{C}\right)}{\left(C^{\gamma \alpha}+\bar{C}\right)}
$$

where $L$ is the semi-extent of the austenite grain. Likewise, the position of the interface $Z_{3}$ when the carbon activity becomes uniform is calculated using the appropriate mass balance, and it is expressed as follows:

$$
Z_{3}=L\left(1-\frac{\bar{C}}{C^{\gamma \alpha}}\right)
$$

On the other hand, the carbon concentration in the center of the austenite grain $\left(C_{L}\right)$ can also be calculated also by balancing the amount of carbon enrichment of austenite against the carbon depletion in the ferrite at an intermediate position $Z_{2}$ (Figure 6(b)) during the soft impingement process $\left(Z_{1}<Z_{2}<Z_{3}\right)$,

$$
C_{L}=\frac{2 L \bar{C}-C^{\gamma \alpha} \cdot\left(L-Z_{2}\right)}{L-Z_{2}}
$$

The instantaneous interfacial carbon mass balance is described as follows: 


$$
C^{\gamma \alpha} \frac{d Z_{2}}{d t}=-D_{C}^{\gamma} \frac{d C}{d z}
$$

where $\frac{d C}{d z}$ represents the gradient of carbon ahead of the interface. Figure 6(b) shows that, in position $z=Z_{2}$, this gradient can be expressed by:

$$
\frac{d C}{d z}=-\frac{C^{\gamma \alpha}-C_{L}}{L-Z_{2}}
$$

Finally, combining Eqn. (7), (8), and (9) the following differential equation is obtained:

$$
\frac{d Z_{2}}{d t}=\frac{2 D_{C}^{\gamma}\left(Z_{3}-Z_{2}\right)}{\left(L-Z_{2}\right)^{2}}
$$

This velocity expression may then be integrated to yield the interface position (i.e. thickness of ferrite) as a function of time during isothermal decomposition of austenite. In one-dimensional model the calculated volume fraction of allotriomorphic ferrite can be then approximated as:

$$
V_{\alpha}=\frac{Z}{L} ; \quad\left(z=Z_{2}\right)
$$

According to Eqns (6) and (11) with $z=Z_{3}$, a maximum volume fraction of $26 \%$ is obtained during the isothermal decomposition of austenite into ferrite at $973 \mathrm{~K}$, which is in excellent agreement with the experimental value listed in Table II. Figure 7 shows the calculated and measured $V_{\alpha}$ values under parabolic and soft impingement growth conditions. This figure 
suggests that care needs to be taken with the assumption of semi-infinite austenite grain extent and the modeling of allotriomorphic ferrite transformation under parabolic growth conditions in medium carbon microalloyed steels. It can be concluded that the soft impingement effect should be considered in the study of allotriomorphic ferrite growth kinetics of this steel. From Figure 8 it is concluded that a great overall level of agreement between experiment and theory exists as soft impingement growth conditions are considered in calculations. Points lying on the line of unit slope show a perfect agreement between experimental and calculated values. $\mathrm{R}^{2}$ in Figure 8 is the square correlation factor of the experimental and calculated volume fraction of ferrite and quantifies the accuracy of the calculations. The accuracy of the model is $98 \%$, which can be considered excellent for a thermodynamic and kinetics model.

\section{Kinetics of allotriomorphic ferrite formation at $T<A e_{1}$}

At temperatures lower than $A e_{1}$, the isothermal decomposition of austenite yields to a ferritepearlite final microstructure. Pearlite is a lamellar product of eutectoid decomposition. A pearlite nodule is composed of multiple colonies; each colony has parallel lamellae of ferrite and cementite, which are orientated differently with respect to lamellae in adjacent colonies. The austenite transforms into pearlite by a reconstructive mechanism at temperatures below the eutectoid temperature $A e_{1}$. The formation of allotriomorphic ferrite enriches in carbon the surrounding austenite promoting the formation of cementite nucleus at the $\alpha / \gamma$ interface and the local reduction of carbon content in the austenite that surrounds the cementite nucleus leads to the ferrite formation of pearlite aggregate. The simultaneous ferrite and cementite formation process

yields to the characteristic lamellar structure of pearlite ${ }^{[3]}$. As soon as pearlite surrounds a ferrite 
allotriomorph, its growth will finish as it is shown in Figure 9. In addition, the carbon enrichment of austenite due to allotriomorphic ferrite formation is avoided and carbon concentration in austenite far from the $\alpha / \gamma$ interface remains the same as the overall carbon content of the steel. Hence, the soft-impingement effect can be neglected. Allotriomorphic ferrite is considered to grow under a parabolic law, and the assumption of a semi-infinite extent austenite with constant boundary conditions is suitable for the kinetics of the isothermal decomposition of austenite at temperatures below $A e_{1}$.

Reed and Bhadeshia ${ }^{[44]}$ proposed the following equation to describe the evolution of $V_{\alpha}$ with time $(t>\tau)$ during the isothermal decomposition of austenite assuming site saturation for nucleation of ferrite in austenite grain boundaries, and within the framework of Johnson-MehlAvrami heterogeneous transformation kinetics theory:

$$
V_{\alpha}=V_{e}\left[1-\exp \left(-\frac{2 S_{V} \alpha_{1}(t-\tau)^{1 / 2}}{\phi}\right)\right]
$$

where $t$ is the isothermal holding time; $\tau$ is the incubation time calculated according to Eqn. (1); $\alpha_{1}$ is the one-dimensional parabolic rate constant; $S_{V}$ is the austenite grain surface per unit volume; $V_{e}$ is the equilibrium volume fraction of allotriomorphic ferrite and $\phi$ is the supersaturation in carbon which can be estimated from the phase diagram as follows:

$$
\phi=\frac{\bar{C}-C^{\gamma \alpha}}{C^{\alpha \gamma}-C^{\alpha \gamma}}
$$


Assuming austenite grains to be tetrakaidecahedra, $S_{V}$ in Eqn. (12) can be expressed in terms of the average austenite grain diameter $d_{\gamma}$ by ${ }^{[8]}$ :

$$
S_{V}=\frac{3.35}{d_{\gamma}}
$$

with $d_{\gamma}=2 \mathrm{~L}$ (Figure 6(a)).

Figure 10 shows the experimental and predicted evolution of $V_{\alpha}$ during the isothermal decomposition of austenite at 913 and $873 \mathrm{~K}$. Likewise, Figure 11 shows in more detail the $V_{\alpha}$ values corresponding to the beginning of the allotriomorphic ferrite transformation at both temperatures. From both figures it is concluded that the lower ferrite formation temperature, the slower allotriomorphic ferrite grows. That behavior is consistent with the lower temperature, the lower carbon mobility in austenite. A comparison of the calculated and experimental $V_{\alpha}$ values for $\mathrm{T}<A e_{1}$ is shown in Figure 12. This figure suggests that a good agreement $\left(93 \%\right.$ in $\mathrm{R}^{2}$ ) between experiment and theory exists in the calculations.

Finally, it is worthy to mention that this model is based on physical and metallurgical principles of phase transformations. Although the proposed model has only been validated for a 0.37C-1.45Mn-0.11V steel, in principle this model is able to predict the isothermal decomposition of austenite for a wide range of steels. This model is different to those empirical and semiempirical models created by fitting equations to experimental data.

\section{CONCLUSIONS}


1. The kinetics of austenite-to-allotriomorphic ferrite transformation has been described in a wide temperature range for a $0.37 \mathrm{C}-1.45 \mathrm{Mn}-0.11 \mathrm{~V}$ (in wt-\%) microalloyed steel. Since the austenite decomposition products at temperatures above and below $A e_{1}$ are different, two different mathematical models have been proposed for the isothermal austenite decomposition in allotriomorphic ferrite.

2. A mathematical process to calculate the incubation time for the isothermal formation of allotriomorphic ferrite has been described. Experimental validation of calculations has been carried out using dilatometric and metallographic analysis. An excellent agreement between calculated and measured incubation time values for the isothermal formation of allotriomorphic ferrite has been found for the studied steel.

3. Gilmour et al. analysis considering soft-impingement effect gives a very good representation of the kinetics of austenite-to-allotriomorphic ferrite transformation at temperatures above $A e_{1}$ in the studied steel. An excellent agreement (98\% in $\mathrm{R}^{2}$ ) has been obtained between experimental and predicted values of volume fraction of allotriomorphic ferrite.

4. Since pearlite is formed at temperatures below $A e_{1}$, the soft-impingement effect should be neglected for the austenite-to-allotriomorphic ferrite transformation kinetics. In that case, Reed and Bhadeshia analysis considering the growth of allotriomorphic ferrite under a parabolic law, is the most suitable representation of allotriomorphic ferrite transformation kinetics. A very good agreement $\left(93 \%\right.$ in $\left.\mathrm{R}^{2}\right)$ between calculated and measured volume fractions of allotriomorphic ferrite has been found at those temperatures. 


\section{ACKNOWLEDGEMENTS}

The authors acknowledge financial support from the Spanish Comisión Interministerial de

Ciencia y Tecnología (CICYT) (project-PETRI 95-0089-OP). GSB Acero S.A and CEIT are thanked for providing the steel and their collaboration in this project. 


\section{REFERENCES}

1. H.I. Aaronson: (1955) Symposium on the Mechanism of Phase Transformations in Metals, Institute of Metals, London, 1955, p. 47.

2. C. A. Dube, H. I. Aaronson and R. F. Mehl: Revue de Metallurgie, 1958, 3, pp. 201-210.

3. J.W. Christian: Theory of Phase Transformations and Alloys, 2nd edition Pt. 1, Pergamon Press, Oxford, 1975, p. 10.

4. H.K.D.H Bhadeshia: Mathematical Modelling of Weld Phenomena III, H. Cerjak and H.K.D.H. Bhadeshia, Institute of Materials, London, 1996, pp.1-50.

5. H.K.D.H. Bhadeshia, L.E-. Svensson and B. Gretoft: Proc. Conf. On Welding Metallurgy of Structural Steels, J.Y. Koo, TMS-AIME, Warrendale, PA, 1987, pp.517-530.

6. G. P. Krielaart, M. Onik, C.M. Brakman, F.D. Tichelaar, E.J. Mittemeijer and S. van der Zwaag: Zeitschrift für Metallkunde, 1994, 84, pp.756-765.

7. M. Unemoto, A. Hiramatsu, A. Moriya, T. Watanabe, S. Nanaba, N. Nakajima, G. Anan and Y. Higo: ISIJ International, 1992, 32, pp.306-315.

8. J. W. Cahn: Acta Metall., 1956, 4, pp. 449-459.

9. M. Hillert and L. I. Staffanson: Acta Chem. Scand., 1970, 24, pp. 3618-3626.

10. K. L. Lee, J. K. Lee, K. B. Kang and O. Kwon: ISIJ International, 1992, 32, pp. 326-334.

11. Madariaga, I. Gutierrez, C. Garcia de Andres and C. Capdevila: Scripta Metall.et Mater, 1999, 41, p. 229-235.

12. Madariaga and I. Gutierrez: Acta Mater., 1999, 47, pp. 951-960.

13. Madariaga, I. Gutiérrez and J. L. Romero: Metall. Trans. A, 1998, 29A, p. 1003.

14. M. A. Linaza, J. L. Romero, J. M. Rodríguez-Ibabe and J.J. Urcola: Scripta Metall., 1993, 29, p. 1217. 
15. I Madariaga, J. L. Romero and I. Gutiérrez: Metall. Trans. A, 1998, 29A (1998) 1003.

16. C, García De Andrés, C. Capdevila, and F.G. Caballero: Proc. Congreso Nacional de Tratamientos Térmicos y de Superficie TRATERMAT 98, M. Carsi et al., CENIM-CSIC, Madrid, Spain, 1998, p. 135.

17. H.K.D.H. Bhadeshia: Mater. Sci. Technol., 1985, 1, p. 497.

18. S.S. Babu, H.K.D.H. Bhadeshia, and L.E-. Svensson: J. Mater. Sci. Lett., 1991, 10, p. 142.

19. S.S. Babu and H.K.D.H. Bhadeshia: Mater. Sci. Technol., 1990, 6, p. 1005.

20. C. García de Andrés, C. Capdevila, F. G. Caballero and H.K.D.H. Bhadeshia: Scripta Materialia, 1998, 39, pp. 853-859.

21. C. García de Andrés, G. Caruana and L.F. Alvarez: Mater. Sci. Eng., 1998, A241, p. 211.

22. J. Bardford and W.S. Owen: J. Iron Steel Institute, 1961, 197, p. 146.

23. A.K. Sinha: Ferreous Physical Metallurgy, Butterworths, Boston, USA, 1989, p. 379.

24. K.C. Russell: Acta Metall., 1968, 16, p. 761.

25. H.K.D.H Bhadeshia: Metal Sci., 1982, 16, p. 159.

26. G.F. Vander Voort: Metallography. Principles and Practice, McGraw-Hill, New York, 1984, p. 27,

27. C. García, L. F. Alvarez and M. Carsí: Welding International, 1982, 6, p. 612.

28. W.F. Lange, M. Enomoto and H.I. Aaronson: Metall. Trans. A, 1988, 19A, p.427.

29. R.H. Siller and R.B. Mclellan: Metall. Trans. A, 1970, 1, p. 985.

30. H.K.D.H. Bhadeshia: Metal Sci., 1981,15, p. 477.

31. S. M. Hodson: MTDATA-Metallurgical and Thermomechanical Databank, National Physical Laboratory, Teddington, UK, 1989, p. 1

32. H.K.D.H. Bhadeshia: Materials Algorithms Project (MAP), URL: www.msm.cam.ac.uk/map/steel/subs/ferr-b.html. 
33. N. Ridley, H. Stuart and L. Zwell: Trans. AIME, 1969, 245, p. 1834.

34. D.J. Dyson and B. Holmes: JISI, 1970, 208, p. 469.

35. N.A. Gjostein, H.A. Domian, H.I. Aaronson and E. Eichen: Acta Metall., 1966, 14, p. 1637.

36. R.W.K. Honeycombe and H.K.D.H. Bhadeshia: Steels: Microstructure and Properties, Arnold, London, UK, 1995, p. 5.

37. C. Atkinson, H. B. Aaron, K. R. Kinsman and H.I. Aaronson: Metall. Trans. A, 1973, 4, p.783.

38. C. Zener: J. Applied Phys., 1949, 20, p.950.

39. J.W. Christian: Theory of Tranformations in metals and alloys, Part I, 2nd edn, Pergamon , Oxford, UK, 1975, p.482.

40. H.K.D.H. Bhadeshia: Progress in Materials Science, 1985, 29, p. 321.

41. G.J. Shiflet, J.R. Bradley and H.I. Aaronson: Metall. Trans. A, 1978, 9A, p. 999.

42. J.B. Gilmour, G.R. Purdy, and J.S. Kirkaldy: Metall. Trans. A, 1972, 3, p. 3213.

43. C. Capdevila, C. Garcia de Andres and H.K.D.H Bhadeshia: Materials Algorithms Project (MAP), URL: www.msm.cam.ac.uk/map/steel/programs/ferr-b.html

44. R.C. Reed and H.K.D.H. Bhadeshia: Mater. Sci. Technol., 1992, 8, p. 421. 


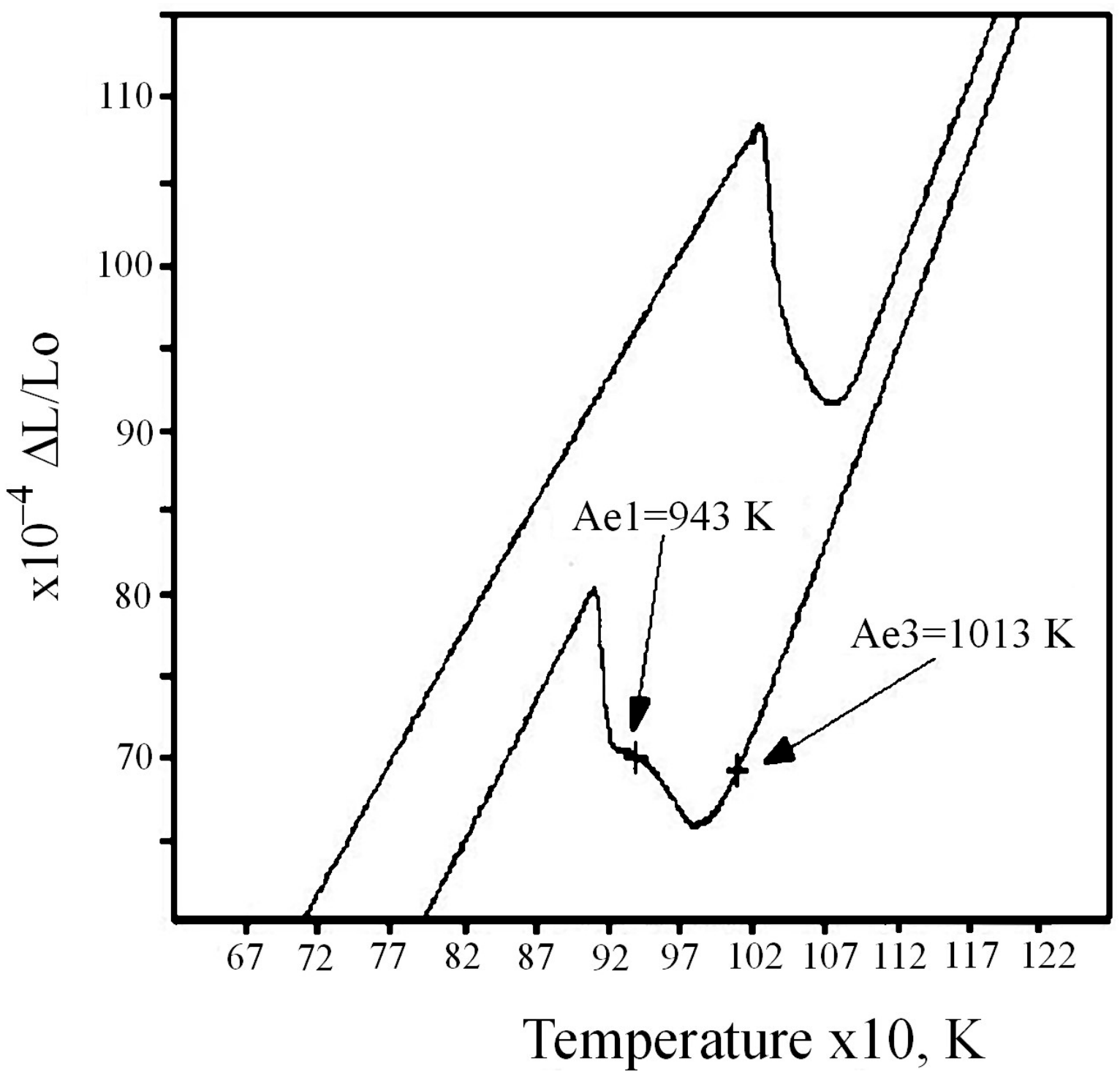

Fig. $1-A e_{3}$ and $A e_{1}$ critical temperatures marked on the cooling segment of a dilatometric curve obtained by continuous cooling at $0.05 \mathrm{~K} / \mathrm{s}$. 


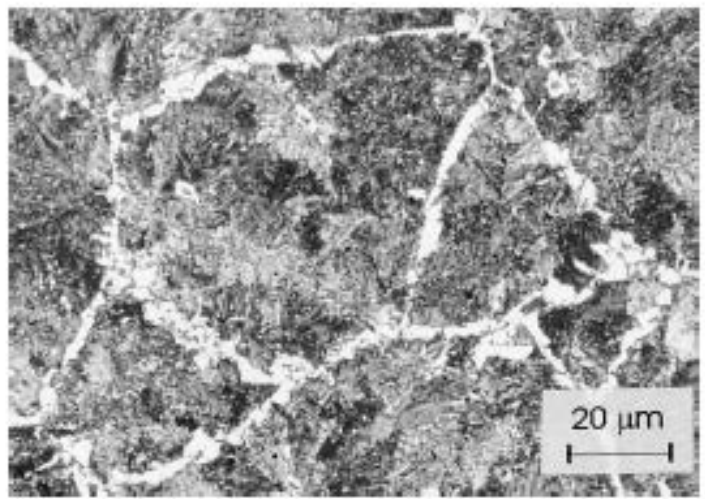

(a)

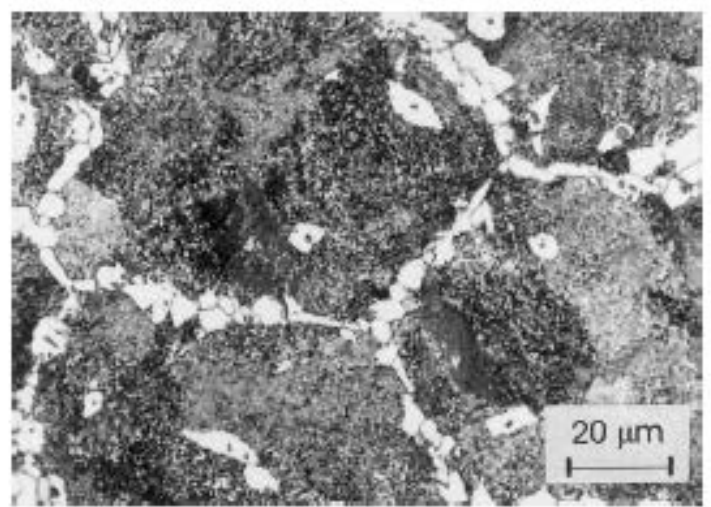

(b)

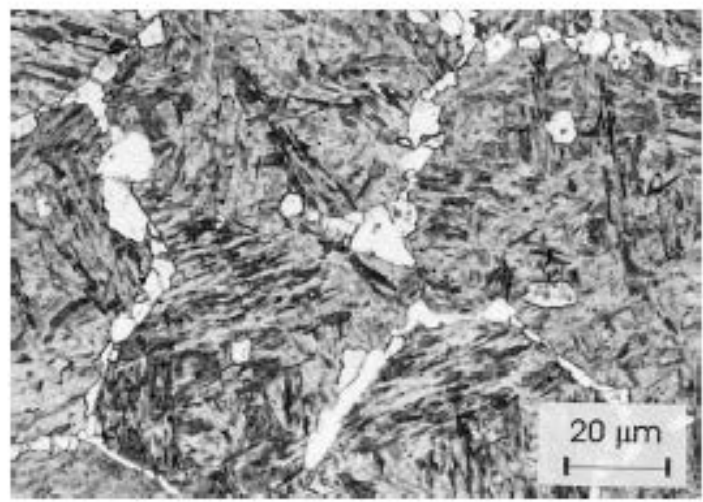

(c)

Fig. 2- Microstructures obtained after isothermal heat treatment during $10 \mathrm{~h}$ at (a) $973 \mathrm{~K}$, (b) $913 \mathrm{~K}$ and (c) $873 \mathrm{~K}$. 


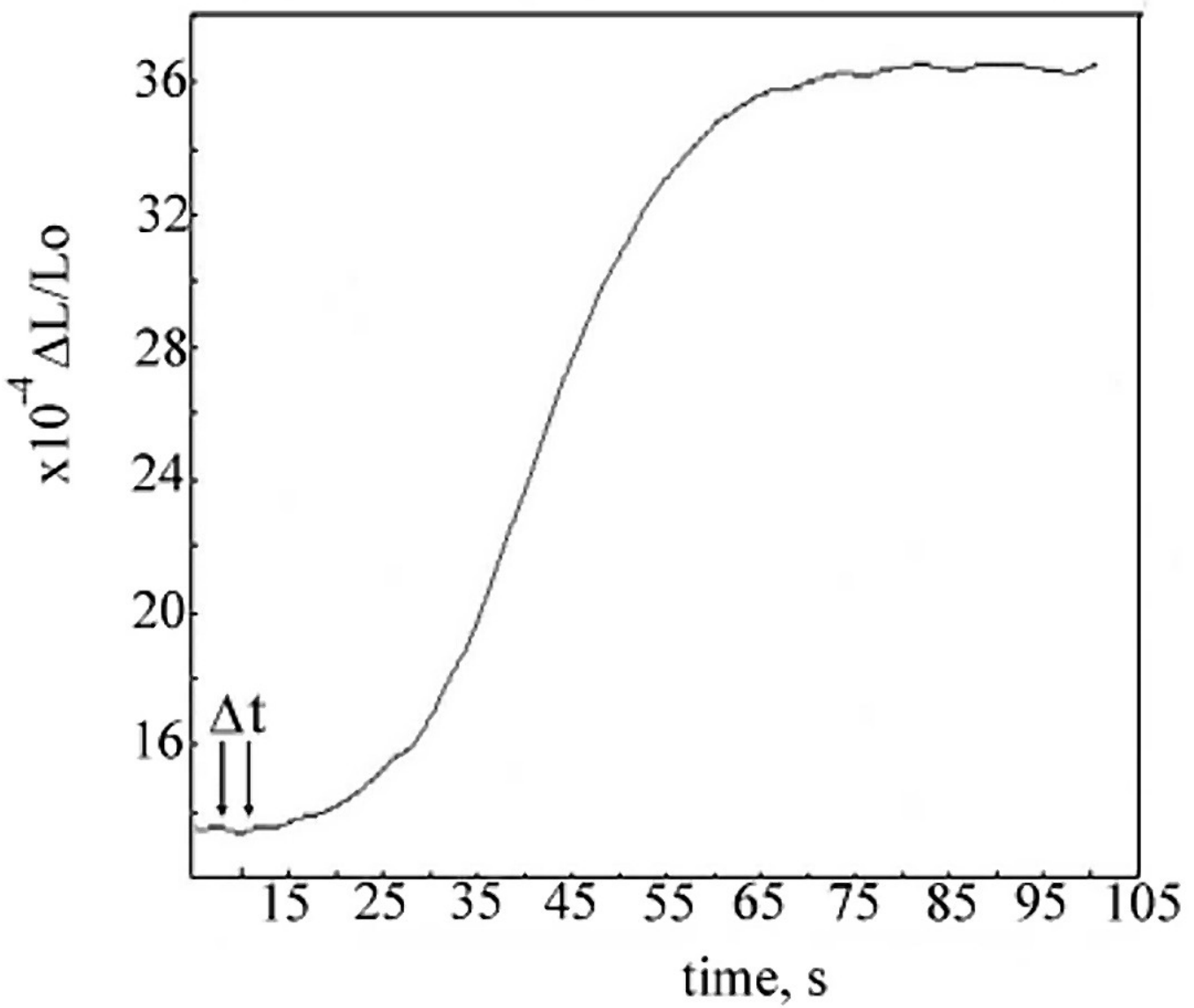

Fig. 3- Dilatometric curve (relative change in length vs. time) obtained during isothermal decomposition of austenite at $873 \mathrm{~K}$ during $100 \mathrm{~s}$ for the studied steel. 


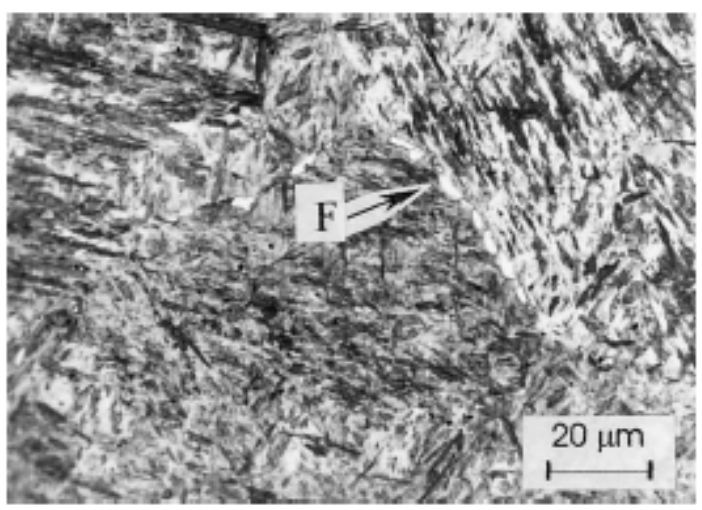

Fig. 4- Optical micrograph of studied steel after isothermal heat treatment at $913 \mathrm{~K}$ during $18 \mathrm{~s}$. 


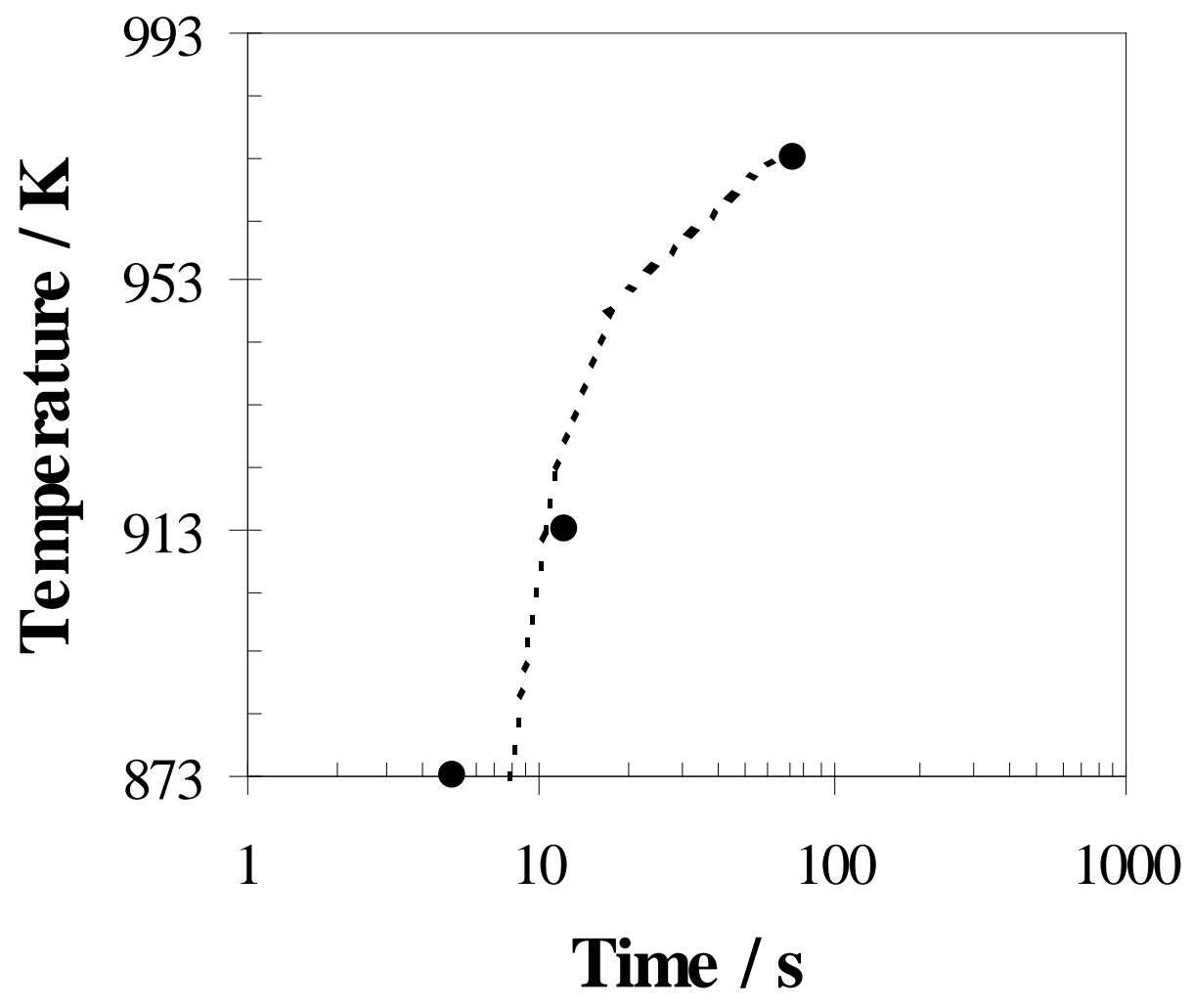

Fig. 5- Experimental and calculated results for the incubation time of isothermally formed allotriomorphic ferrite 


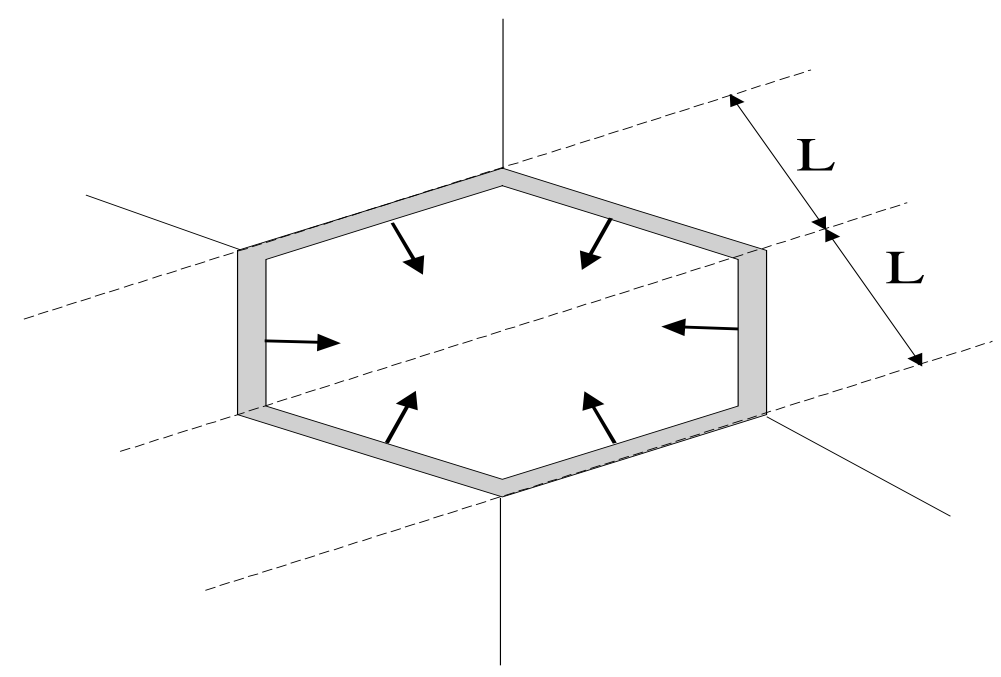

(a)

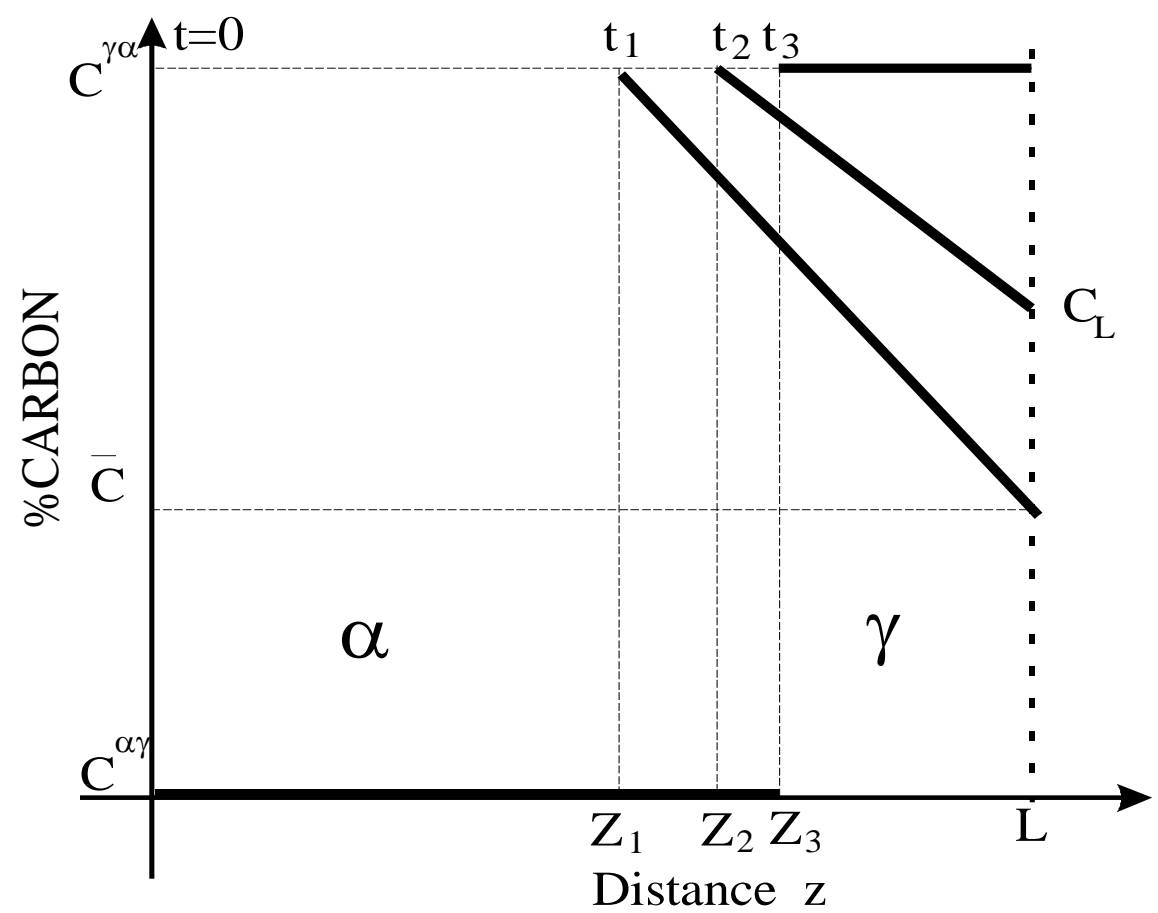

(b)

Fig. 6- Diagram illustrating the soft-impingement process: (a) Schematic representation of the growth of grain boundary allotriomorphs, (b) carbon concentration profile for the calculation of impingement time. The dark lines represent the carbon profile in austenite and ferrite. 


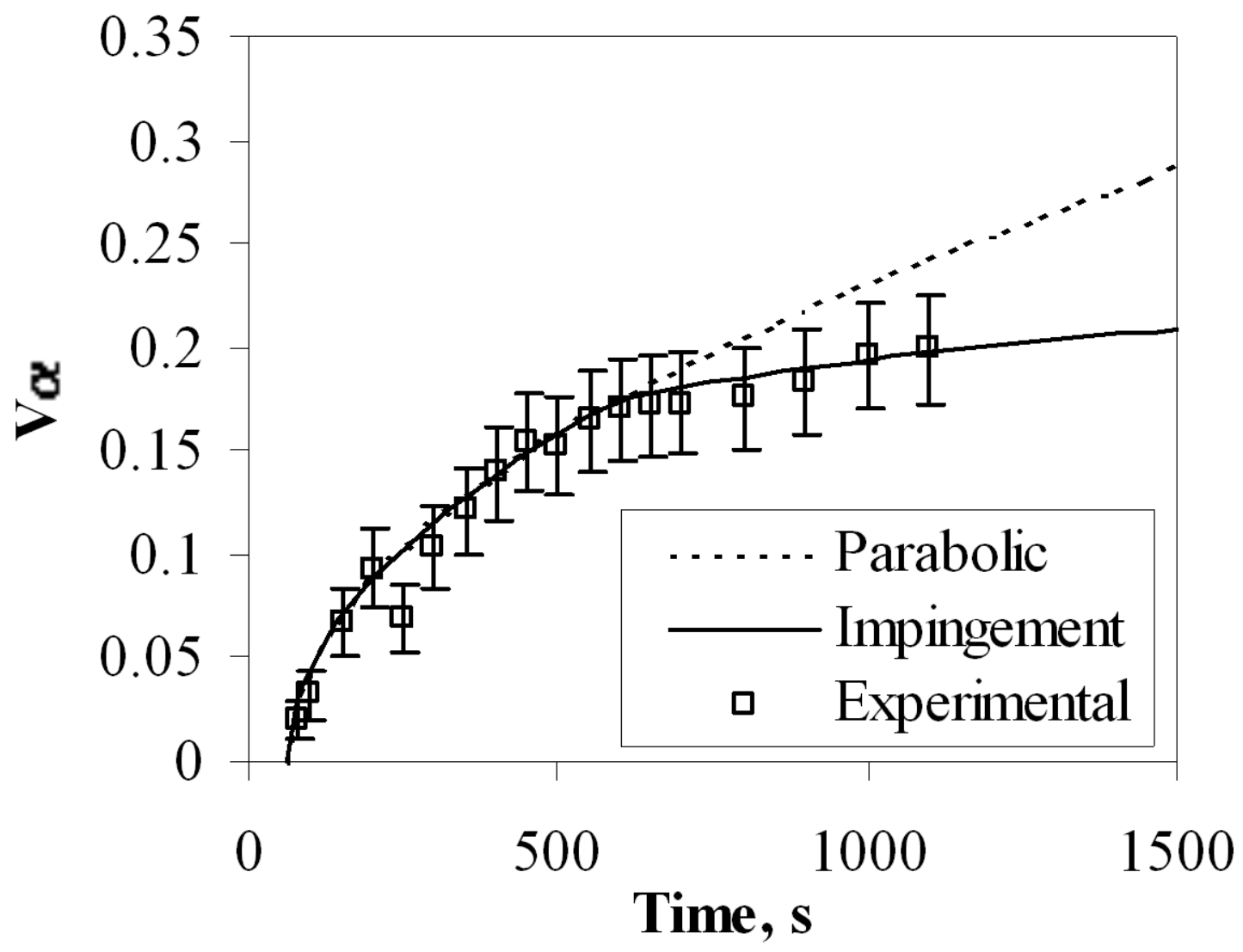

Fig. 7- Comparison between measured and calculated $V_{\alpha}$ values under parabolic and impingement considerations during the isothermal decomposition of austenite at $973 \mathrm{~K}$. 


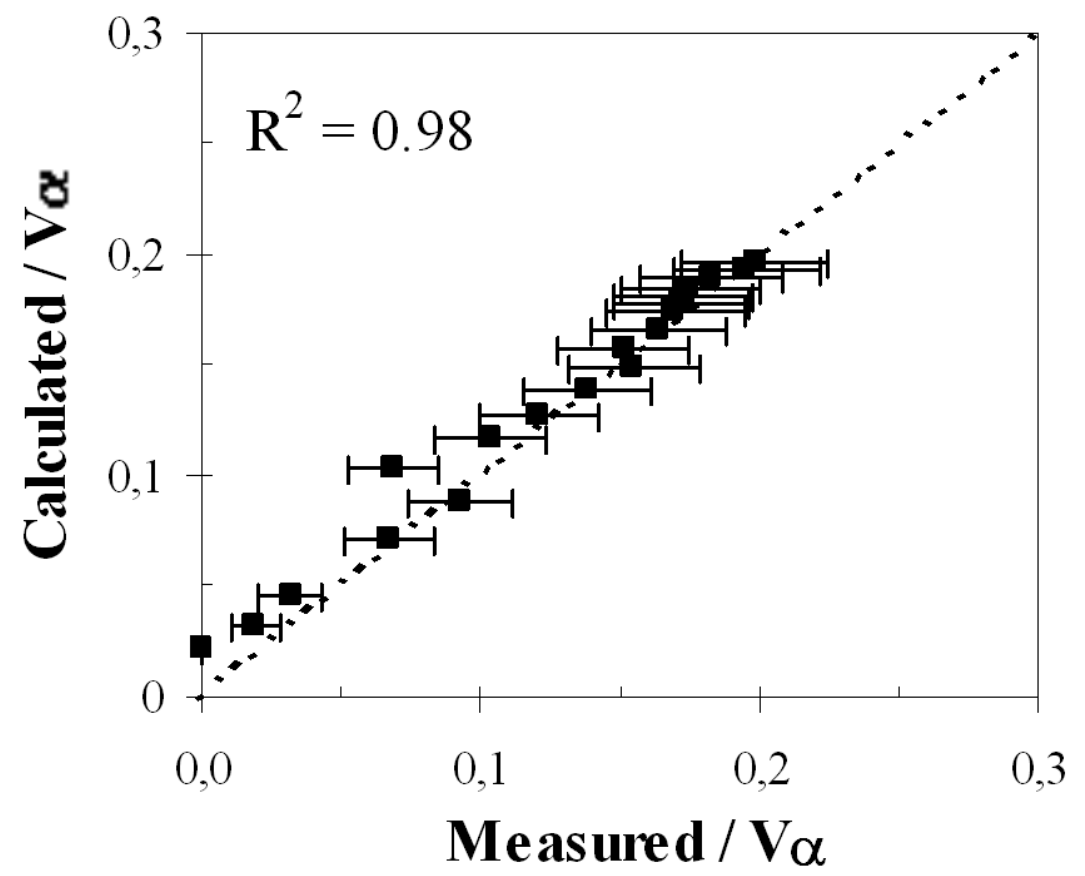

Fig. 8- A comparison of the experimental and predicted ferrite volume fraction formed at a temperature higher than $A e_{1}$. Soft impingement is considered in calculations. 


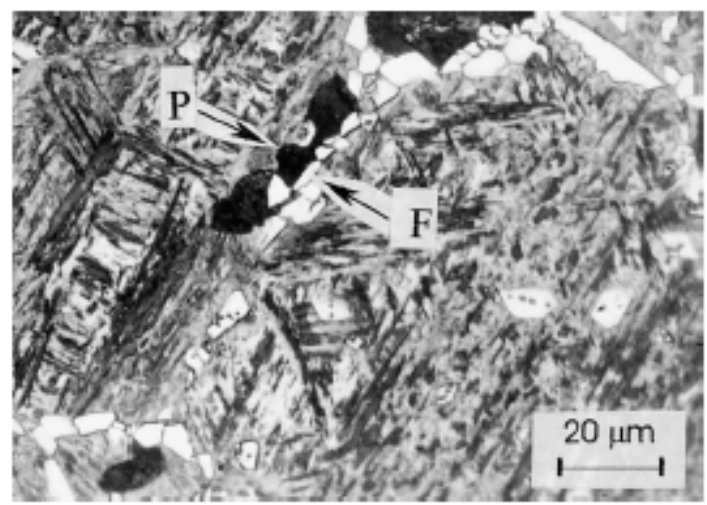

Fig. 9- Early stage of pearlite transformation during the isothermal decomposition of austenite at $913 \mathrm{~K}$ during $60 \mathrm{~s}$ 


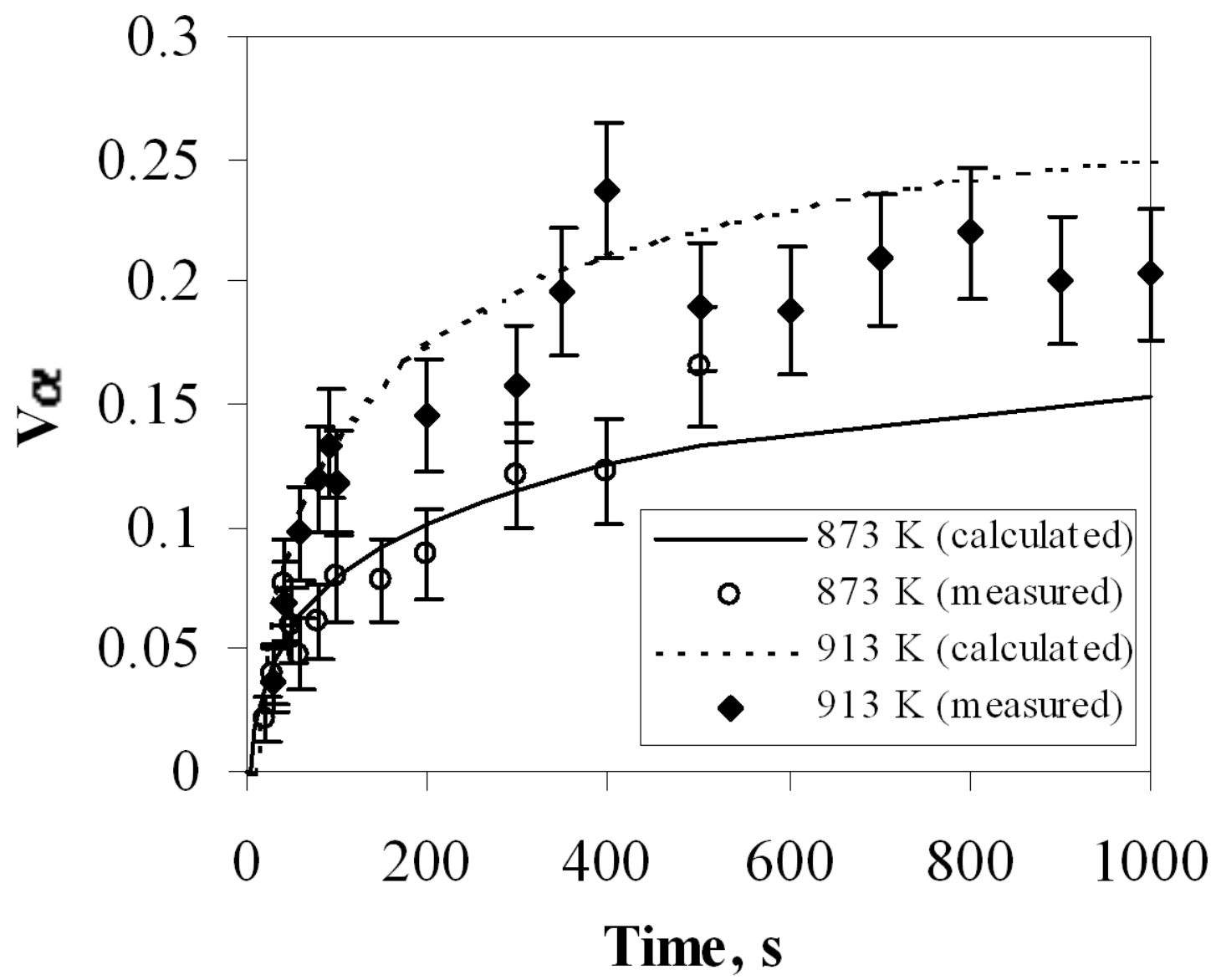

Fig. 10- Comparison between calculated and measured $V_{\alpha}$ values during the isothermal decomposition of austenite at 913 and $873 \mathrm{~K}$. 


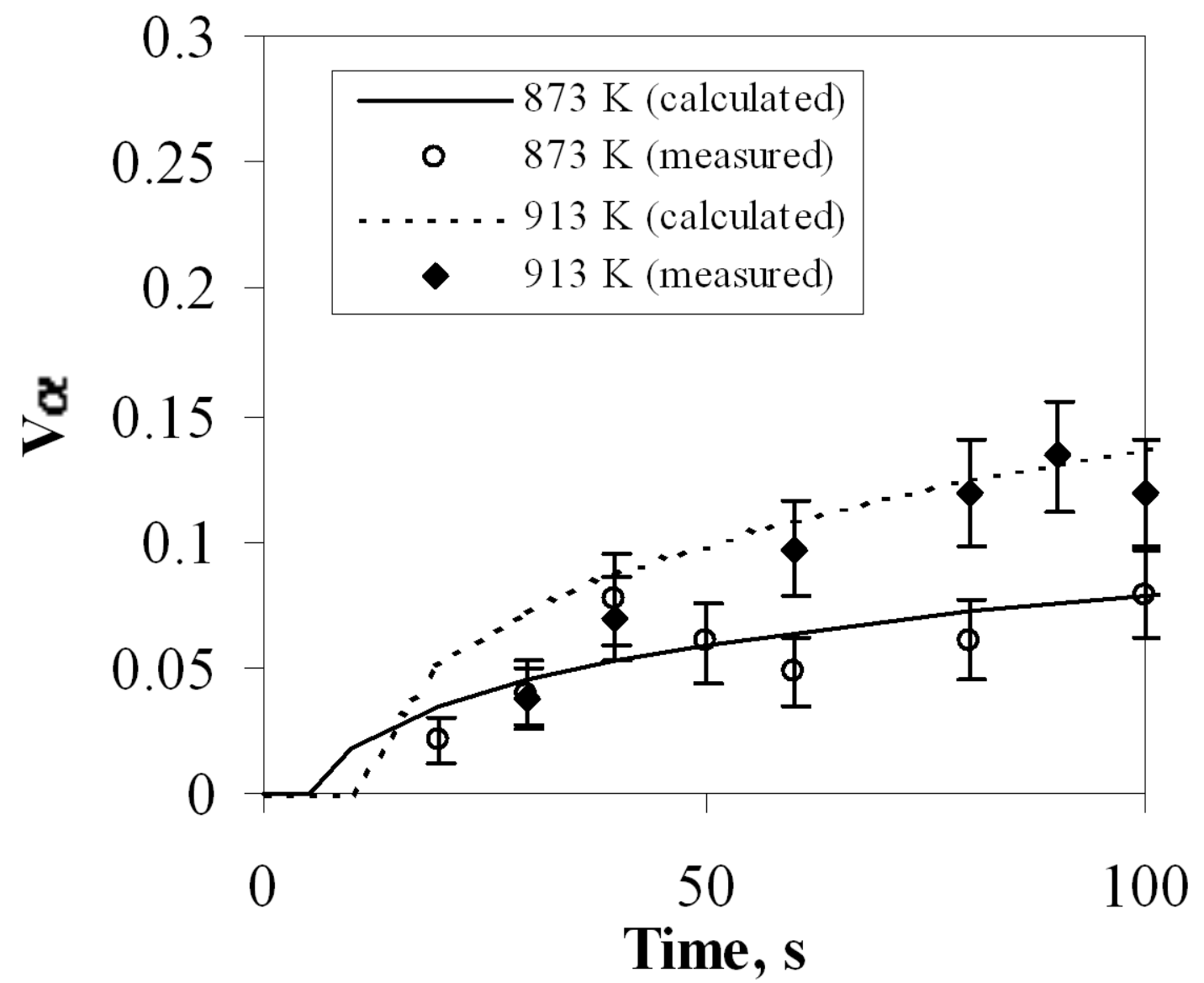

Fig. 11- Comparison between calculated and measured $V_{\alpha}$ values at the initial stages of isothermal decomposition of austenite in allotriomorphic ferrite at 913 and $873 \mathrm{~K}$. 


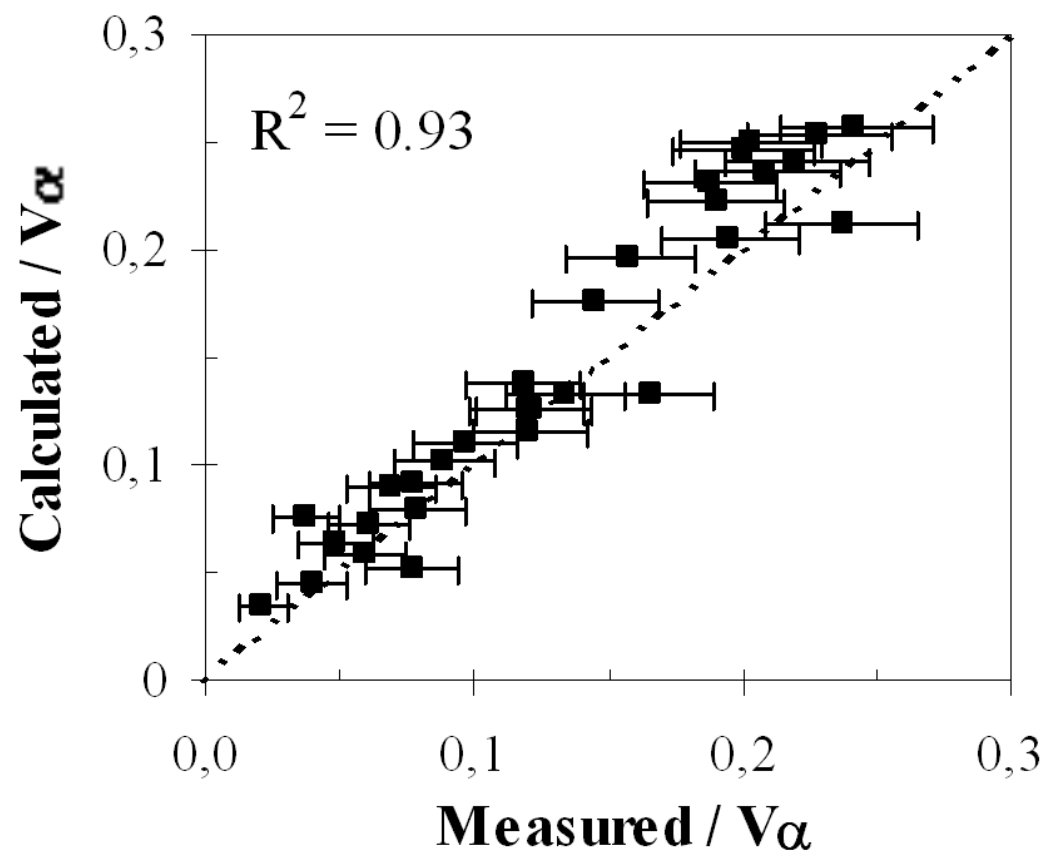

Fig. 12- A comparison of the experimental and predicted ferrite volume fraction formed at a temperature lower than $A e_{1}$. 